\title{
Hourly Global Solar Irradiation Forecasting for New Zealand
}

\author{
A. Ahmad*, T.N. Anderson and T.T. Lie \\ School of Engineering, Auckland University of Technology, Auckland 1142, New Zealand
}

\begin{abstract}
With the growing use of solar thermal energy systems and small scale photovoltaic power generation by domestic users, there is increasing need to develop intelligent controllers that allow these users to efficiently manage the energy generated by these systems. Ideally these intelligent controllers will be able to forecast the availability and magnitude of the solar resource to plan in advance for periods when the solar irradiance magnitude is small or unavailable. In addition, the method used to provide this forecast needs to be adaptable to a range of timescales and locations. With this in mind, this study examined the possibility of providing a 24-hour ahead forecast of hourly global solar irradiation in New Zealand using several approaches but with particular reference to nonlinear autoregressive recurrent neural networks with exogenous inputs (NARX).
\end{abstract}

Hourly time series data for nine historic weather variables recorded over a three year period was used to train and test the forecasting methods for New Zealand's largest city, Auckland. Results from forecasts based on the NARX were compared with an artificial neural network (ANN) based Multilayer Perceptron (MLP) method, a statistical approach using auto regressive moving average (ARMA) and a reference persistence approach. Predicted values of hourly global solar irradiation were compared with the measured values, and it was found that the root mean squared error (RMSE) was $0.243 \mathrm{MJ} / \mathrm{m}^{2}$ for the NARX method as compared to $0.484 \mathrm{MJ} / \mathrm{m}^{2}, 0.315 \mathrm{MJ} / \mathrm{m}^{2}$ and $0.514 \mathrm{MJ} / \mathrm{m}^{2}$ for the MLP, ARMA and persistence approaches respectively. Subsequently the NARX approach was used to forecast global solar irradiation for other major cities across New Zealand. The results demonstrate the ability of the NARX approach to forecast irradiation values at a later time and across a number of different locations. As such it is foreseeable that such an approach could serve as the basis of a forecasting system in future intelligent controllers.

Keywords: Solar Irradiation Forecasting; NARX ANN; Multilayer Perceptron; Auto Regressive Moving Average

\footnotetext{
* Corresponding Author: Tel.: +64 99219999 Ext 8109

E-mail address: aahmad@aut.ac.nz
} 


\section{Introduction}

Building and operating reliable solar energy systems requires information about global solar irradiation in the region where the system is. To best manage the energy generated by these systems, the solar irradiation has to be predicted accurately for their location. This can be achieved using various solar irradiation estimation techniques that can help efficiently optimize energy distribution between loads and the local grid.

In many cases limited coverage of irradiation measuring networks prompts the development of techniques for estimating and forecasting the global irradiation using climatological parameters (Maitha, et al., 2011). Several techniques have been developed in order to estimate solar irradiance data at different scales including empirical (Loutzenhier et al., 2007), analytical (Ulgen and Hepbasli, 2009) and numerical techniques, as well as neural network approaches (Moustrisa et al., 2008). Additionally, a significant number of studies have used physics-based approaches as shown by Gueymard, (2005, 2008), and Perez at al., (2007) and also statistical forecasting of solar irradiation (Goh and Tan, 1977).

Approaches based on statistical processes such as autoregressive (AR), moving-average (MA), autoregressive moving-average (ARMA), autoregressive-integrated moving-average (ARIMA) and Markov chain have also been widely used. However, these approaches require some statistical transformations to the data before they are applied. Due to these transformations, it is not possible to ensure that the results are accurate and represent a precise correlation with the measured solar irradiance values (Al-Alawi and Al-Hinai, 1998) and so it is necessary to develop alternative approaches (Mellit et al., 2010).

In this regard, artificial neural network (ANN) techniques for predicting irradiation have been shown to have greater accuracy than other techniques such as linear, nonlinear and fuzzy approaches (Yadav and Chandel, 2014) and so have been used in a number of solar energy applications. Kalogirou, (2001) has reviewed the use of ANN in renewable energy systems applications while Mellit and Kalogirou, (2008) and Mellit et al. (2009) reviewed ANN's in photovoltaic applications and for sizing of photovoltaic systems respectively. Similarly authors such as Esen et al. (2008) have examined adaptive neuro-fuzzy inference systems (ANFIS) and ANN models of ground-coupled heat pump (GCHP) systems.

Based on the predictive capabilities of ANN systems, a number of studies have begun to examine the ability of these systems to forecast future values of solar irradiation. Mellit, (2008) presented 
a review of artificial intelligence techniques for solar radiation forecasting and concluded that ANN models can be generalized to be used in different locations around the world.

In an early study in this area Sfetsos and Coonick, (2000) introduced an approach for a single step ahead prediction of mean hourly solar radiation received by a horizontal surface through ANN and ANFIS models. It was shown that the performance of the models was enhanced when a wind direction term was included in the input list. They also found that the best prediction resulted from the use of a multivariate Levenberg Marquardt (LM) case that exhibited a 74\% improvement in the Root Mean Square error when compared with a persistence approach. The results indicated that the ANN models predict the solar radiation time series more effectively than procedures based on the clearness index.

Christophe et al. (2010) also used an ANN prediction approach based on Multi-Layer Perceptron to determine global irradiation at daily horizon $(d+1)$. Their proposed model was compared with AR, ARMA, k-Nearest Neighbors (k-NN) and Markov Chains approaches. Without preprocessing AR and ANN models showed a daily normalised Root Mean Square Error (nRMSE) of approximately $21 \%$ compared to Markov chain, Bayes and k-NN methods where nRMSE was in the order of $25-26 \%$.

More recently, Voyant et al. (2014) developed an ANN based MLP model that was applied to two years of pre-treated time series data in order to forecast global solar irradiation 24-hours ahead. The results of the MLP were compared with those of ARMA and persistence approaches and showed that the prediction error could be reduced when compared to ARMA the persistence approach.

Similarly, Rich et al. (2013) developed statistical models, ANN models, satellite imaging based models, numerical based models and hybrid methods for solar irradiance forecasting. In their work, they found that regressive methods such as AR, MA, ARMA and ARIMA take advantage of the correlated nature of the irradiance observations and tend to work well in both data-poor and data-rich environments. However, it was concluded that ANN modelling offers improved nonlinear approximator performance and provides an alternative approach to physical modelling for irradiance data when enough historical data is available and are not typically temporally limited. 
Now, it is noted in the literature that physics-based, statistical and ANN based feed-forward network techniques have demonstrated their ability to predict future values of solar irradiation, however, recurrent neural network based methods have received little attention. Therefore this study aims to investigate several techniques for forecasting solar irradiation that could be implemented into future solar control systems, and particularly, the ability of nonlinear autoregressive recurrent neural networks to forecast global solar irradiation 24 hours in advance for a number of major New Zealand cities, as shown in Figure 1.

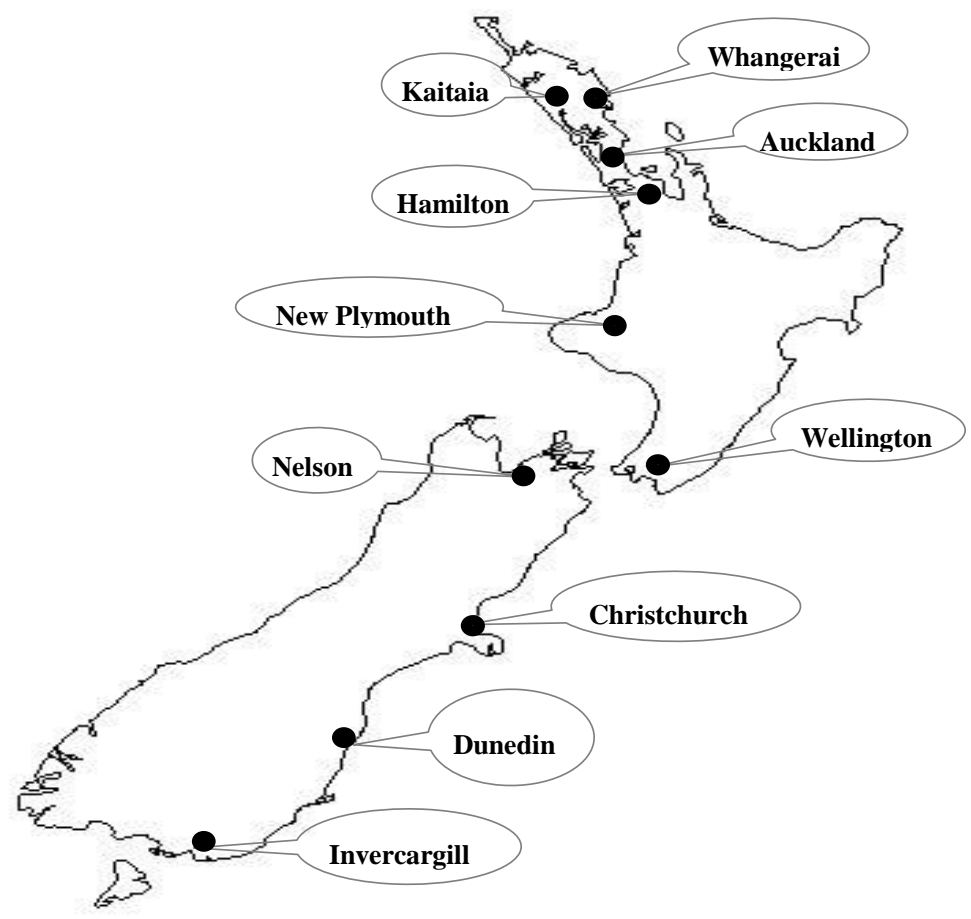

Figure 1. Map of New Zealand with major cities.

\section{Methodology}

For this work several forecasting techniques were utilised: NARX, MLP, ARMA and persistence. However, given the lack of attention it has received in the literature, the NARX ANN architecture is the main typology described in this paper.

For each approach mentioned in this study, three years of historic hourly data for: Temperature $\left(\mathrm{T}_{\max }, \mathrm{T}_{\min }\right)$, Barometric Pressure $(\mathrm{P})$, Relative Humidity $(\mathrm{RH})$, Solar Zenith Angle (SZA), Azimuth Angle $\left(\mathrm{A}_{\mathrm{z}}\right)$, Rain amount $\left(\mathrm{R}_{\mathrm{a}}\right)$, Wind speed $\left(\mathrm{W}_{\mathrm{s}}\right)$ and Wind direction $\left(\mathrm{W}_{\mathrm{d}}\right)$ were taken from the National Institute of Water and Atmosphere's (NIWA) CliFlo database (2014) to test each method, with hourly global solar irradiation 24 hours in advance being the target output variable. 
For the ANN approaches, that is NARX and MLP, the input weather data was presented as unprocessed data, to study the effect of real input variables on the predicted values of the target variable. In doing this $70 \%$ of the historic data was presented to the network during training, allowing the network to be adjusted according to its error. Subsequently $15 \%$ of the data was used to measure network generalization, and to halt training when generalization stops improving. Finally, the remaining $15 \%$ of the data was used to test the network thus providing an independent measure of the network performance during and after training.

For the ARMA and persistence approach, the same data was utilised in developing the forecast, however, a more detailed account of these methods is provided in the latter discussion of each of these.

\subsection{Input variables selection for all prediction methods}

The prediction accuracy of the proposed methods is dependent on the combination of weather predictor variables and training algorithm (Yadav and Chandel, 2014). This is particularly pertinent, as one of the key tasks in time series prediction is the selection of the input variables and the choice of variables depends on data availability, its quality and their correlation. To provide a sound analytical basis for the choice of variables, a statistical analysis was carried out to check the correlation of each input variable with global solar irradiance. The Pearson correlation coefficient $(\mathrm{R})$ is a measure of the linear correlation between two variables, giving a value between +1 and -1 inclusive, where 1 is total positive correlation, 0 is no correlation, and -1 is total negative correlation. The $\mathrm{R}$ values for each input variable with respect to global solar irradiance is shown in Table 1.

Table 1: Regression values for input weather variables vs global solar irradiance

\begin{tabular}{|l|c|}
\hline \multicolumn{1}{|c|}{ Input variables } & R \\
\hline Max Temperature $\left(\mathrm{T}_{\max }\right)$ & 0.462 \\
\hline Min Temperature $\left(\mathrm{T}_{\min }\right)$ & 0.404 \\
\hline Relative Humidity $(\mathrm{RH})$ & 0.505 \\
\hline Rain Amount $\left(\mathrm{R}_{\mathrm{a}}\right)$ & 0.055 \\
\hline Solar Zenith Angle $(\mathrm{SZA})$ & 0.066 \\
\hline Azimuth $\left(\mathrm{A}_{\mathrm{z}}\right)$ & 0.183 \\
\hline Pressure $(\mathrm{P})$ & 0.020 \\
\hline Wind Speed $\left(\mathrm{W}_{\mathrm{s}}\right)$ & 0.002 \\
\hline Wind Direction $\left(\mathrm{W}_{\mathrm{d}}\right)$ & 0.005 \\
\hline
\end{tabular}

From Table 1 it can be seen that $\mathrm{RH}, \mathrm{T}_{\max }$ and $\mathrm{T}_{\min }$ have a reasonable degree of correlation with solar irradiance, while $\mathrm{W}_{\mathrm{s}}$ and $\mathrm{W}_{\mathrm{d}}$ have almost no correlation. To group the input weather variables, Moody et al. (1995), two-step sensitivity analysis technique was utilized. Once the most 
significant variables were determined (based on the regression values), twelve significant combinations of the nine weather predictor variables as shown in Table 2 were tested in order to investigate their effect on the accuracy of the global solar irradiation forecast for Auckland.

Table 2: Test cases based on different combinations of input variables.

\begin{tabular}{|l|l|l|l|}
\hline Case & \multicolumn{1}{|c|}{ Input Parameters } & \multicolumn{1}{|c|}{ Case } & \multicolumn{1}{|c|}{ Input Parameters } \\
\hline 1 & $\mathrm{~T}_{\max }, \mathrm{T}_{\min }, \mathrm{P}, \mathrm{RH}, \mathrm{SZA}, \mathrm{Az}, \mathrm{Ra}, \mathrm{Ws}, \mathrm{Wd}$ & 7 & $\mathrm{P}, \mathrm{RH}, \mathrm{SZA}, \mathrm{Az}, \mathrm{Ra}$ \\
\hline 2 & $\mathrm{~T}_{\max }, \mathrm{T}_{\min }, \mathrm{P}, \mathrm{RH}, \mathrm{SZA}, \mathrm{Az}, \mathrm{Ra}$ & 8 & $\mathrm{~T}_{\max }, \mathrm{T}_{\min }, \mathrm{SZA}$ \\
\hline 3 & $\mathrm{~T}_{\max }, \mathrm{T}_{\min }, \mathrm{P}, \mathrm{RH}, \mathrm{SZA}, \mathrm{Az}$ & 9 & $\mathrm{~T}_{\max }, \mathrm{T}_{\min }, \mathrm{P}, \mathrm{SZA}, \mathrm{Az}$ \\
\hline 4 & $\mathrm{P}, \mathrm{RH}, \mathrm{SZA}, \mathrm{Az}, \mathrm{Ra}, \mathrm{Ws}, \mathrm{Wd}$ & 10 & $\mathrm{RH}, \mathrm{SZA}, \mathrm{Az}, \mathrm{Ra}$ \\
\hline 5 & $\mathrm{~T}_{\max }, \mathrm{T}_{\min }, \mathrm{P}, \mathrm{RH}, \mathrm{SZA}$ & 11 & $\mathrm{~T}_{\max }, \mathrm{T}_{\min }, \mathrm{P}$ \\
\hline 6 & $\mathrm{~T}_{\max }, \mathrm{T}_{\min }, \mathrm{SZA}, \mathrm{Az}$ & 12 & $\mathrm{~T}_{\max }, \mathrm{T}_{\min }, \mathrm{Ws}, \mathrm{Wd}$ \\
\hline
\end{tabular}

\subsection{Nonlinear Autoregressive with exogenous input (NARX)}

Having determined the input variables, a NARX recurrent neural network was developed to forecast future values of global solar irradiation in Auckland, based on the previous values of global solar irradiation and the nine weather predictor variables described. The approach can be expressed mathematically by predicting future values of the solar irradiation time series $y(t)$ from past values of that time series and past values of the weather predictor variables time series $x(t)$. This NARX approach is based on the linear ARX approach that is commonly used in time-series modelling and can be represented by Equation Error! Reference source not found., where the next value of the dependent output signal (global solar irradiation) $y(t)$ is regressed on previous values of the output signal and previous values of an independent input signal (historic weather data).

$$
y(t)=f\left(y(t-24), y(t-25), \ldots, y\left(t-n_{y}\right), x(t-24), x(t-25), \ldots, x\left(t-n_{x}\right)\right)
$$

The NARX approach is implemented using a feed-forward neural network to approximate the function $f$. A diagram of the resulting network is shown in Figure 2, where the $y(t)$ output series is predicted from past values of $y(t)$ and another input series $x(t)$.

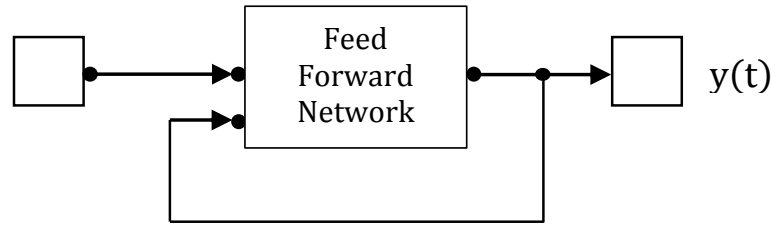

Figure 2: NARX block diagram

There are different connection styles and learning algorithms in neural networks, the most common being the back propagation algorithm. The back propagation algorithm consists of two 
phases: a training phase and recall phase (Fatih et al. 2008). Before the training phase starts, the weights of the network are randomly initialized. Then the output of the network is calculated and compared to the desired value. At each step during training, error of the network is calculated by means of gradient methods and used to adjust the weights of the output layer (Haykin, 1998). In the case of more than one network layer the error is propagated backward to adjust the weights of the previous layers. Once the weights are determined, after several training steps and correlation between different combinations of input variables with targets are finalized, the recall phase may run. In doing this the network output computations are performed using finalized iterations of input data and weights from the training phase.

The training phase is important as it determines the success of the resulting network. In back propagation, there are two methods of updating the weights. In the first method, weights are updated for each of the input patterns using an iteration method. In the second method, used in this study, the mean value of input and output patterns of the training sets is calculated (Yousefizadeh and Zilouchian, 2001). As soon as the weight update values are obtained, the new weights and biases can be calculated using Equation 2.

$$
W_{i j, n}=U_{n}+\alpha W_{i j, n}-1
$$

where $W_{i j, n}$ is a vector of current weights and biases, $\alpha$ is the momentum factor rate which determines how the past weights will reflect to the current value, and $U_{n}$ is the update function which can be chosen according to the problem and data type.

According to Fatih et al. (2008) and Yousefizadeh and Zilouchian, (2001) the most commonly used equation solving algorithm is the LM algorithm. It can be considered as an alternative to the conjugate methods for second derivative optimization. In LM, the update function, $U_{n}$ can be calculated using Equation 3.

$$
U_{n}=-\left[J^{T} \times J+\mu I\right]^{-1} \times J^{T} \times e
$$

where $J$ is the Jacobian matrix that contains the first derivatives of the network errors with respect to the weights and biases, and $e$ is a vector of network errors. The parameter $\mu$ is a scalar number and $I$ is the identity matrix. Depending on when the $\mu$ parameter is large, the update function $U_{n}$ becomes identical to the basic back propagation (with a small step size). During processing the $\mu$ value decreases after each successful step and should be increased only when a tentative step increases the error term or performance function. Consequently the performance function is guaranteed to reduce or to become bounded at each iteration (Martin and Mohammad, 1994). 
In order to determine the performance of developed ANNs quantitatively, and verify whether there was any underlying trend in performance of the ANNs, the regression $(\mathrm{R})$ and root mean squared error (RMSE) values were analysed. The root mean squared error (Equation 4) provides information on the short term performance and is a measure of the variation of predicated values around the measured data, where the lower the RMSE, the more accurate is the estimation.

$$
\text { RMSE }=\sqrt{\frac{1}{N} \sum_{i=1}^{N}\left(I_{p}-I_{i}\right)^{2}}
$$

where $I_{p}$ is the predicted solar irradiance in $M J / \mathrm{m}^{2}, I_{i}$ is the measured solar irradiance in $M J / \mathrm{m}^{2}$, and $N$ denotes the number of observations.

\subsubsection{Number of Hidden Neurons and Delays}

Now in developing a NARX ANN there is an important trade-off to be made between the size and the predictive capability of the network. If the number of neurons or number of delays is increased, the network has the tendency to overfit the data and allows the network to solve more complicated problems but on the other hand requires more computation. Therefore the effect of changing the number of neurons in the hidden layer, increasing and decreasing the number of delays was also investigated. During experiments both the number of neurons in the hidden layer and the number of delays in the tapped delay lines were increased until the network performed well in terms of the root mean square error and the regression values. Using tapped delay lines in the network is essential as it stores previous values of $x(t)$ and $y(t)$ sequences. The number of hidden neurons, network delays and time steps for training, validation and test were varied to determine which network exhibited the best performance.

In this respect, an initial network was developed with the minimum number of neurons and this the number was increased until the network performed well in terms of the root mean square error and regression values. During this phase, each proposed configuration was trained multiple times to stabilize the weight initialization process and deliver the best accuracy in the shortest processing time. As such, networks with up to 250 neurons were examined as part of this sensitivity analysis, however, it was found that a network of 90 neurons was more than adequate, as beyond this the computing time of the network increased significantly without significant increase in the accuracy, similarly delays between 1 and 5 were also tested.

Computing time was observed and it was noted that time increased with increasing numbers of neurons or delays. However, after several trials, it was decided that the most suitable network, 
considering accuracy and computing time, had 90 hidden neurons and 2 delays in the tapped delay lines. The reason computing time was closely monitored, was because if the approach was to be implemented on a standalone hardware platform, such as a solar controller, processing power and memory would be limited compared to desktop resources. Using the weather data for Auckland from Case 2 as an example, Table 3 shows the RMSE and R values for various numbers of neurons in the hidden layer.

Table 3: RMSE, R and computing time values for different numbers of neurons and delays using Case 2 weather data

\begin{tabular}{|c|c|c|c|c|}
\hline $\begin{array}{c}\text { Number of } \\
\text { Neurons }\end{array}$ & $\begin{array}{c}\text { Number of } \\
\text { Delays }\end{array}$ & $\begin{array}{c}\text { RMSE } \\
\left(\mathbf{M J} / \mathbf{m}^{2}\right)\end{array}$ & $\mathbf{R}$ & $\begin{array}{c}\text { Computing Time } \\
(\mathbf{m i n}: \mathbf{s e c})\end{array}$ \\
\hline 5 & 2 & 0.287 & 0.950 & $00: 20$ \\
\hline 10 & 2 & 0.277 & 0.951 & $00: 24$ \\
\hline 20 & 2 & 0.274 & 0.952 & $00: 22$ \\
\hline 30 & 2 & 0.266 & 0.955 & $00: 48$ \\
\hline 40 & 2 & 0.257 & 0.957 & $00: 53$ \\
\hline 50 & 2 & 0.263 & 0.956 & $00: 50$ \\
\hline 50 & 3 & 0.249 & 0.964 & $01: 02$ \\
\hline 90 & 2 & 0.243 & 0.963 & $01: 20$ \\
\hline 90 & 3 & 0.232 & 0.966 & $02: 20$ \\
\hline 90 & 5 & 0.221 & 0.969 & $04: 42$ \\
\hline 150 & 2 & 0.251 & 0.963 & $02: 02$ \\
\hline 200 & 2 & 0.251 & 0.963 & $04: 33$ \\
\hline 250 & 2 & 0.247 & 0.964 & $05: 10$ \\
\hline
\end{tabular}

\subsubsection{NARX Results}

To validate the approach used, the root mean squared error (RMSE) performance function was examined during the training phase. Network training could be stopped early by the validation vectors if the network performance on the validation vectors failed to improve or remained the same, as indicated by an increase in the root mean square error of the validation samples. Test vectors were used as a further check that the network was generalizing well, but did not have any effect on training. The best validation performance for the Auckland weather data shown in Case 2 was $0.2699 \mathrm{MJ} / \mathrm{m}^{2}$ at epoch 9 (a measure of the number of times all of the training vectors are used once to update the weights) with seven input variables.

Further, the network outputs with respect to the target for training, validation, and test sets are shown in Figure 3. The dashed line in each axis represents the perfect result, that is: outputs = targets. The solid line represents linear best fit between the outputs and targets. For this problem, the fit is reasonably good for all data sets, with the overall regression values as high as 0.963 . 

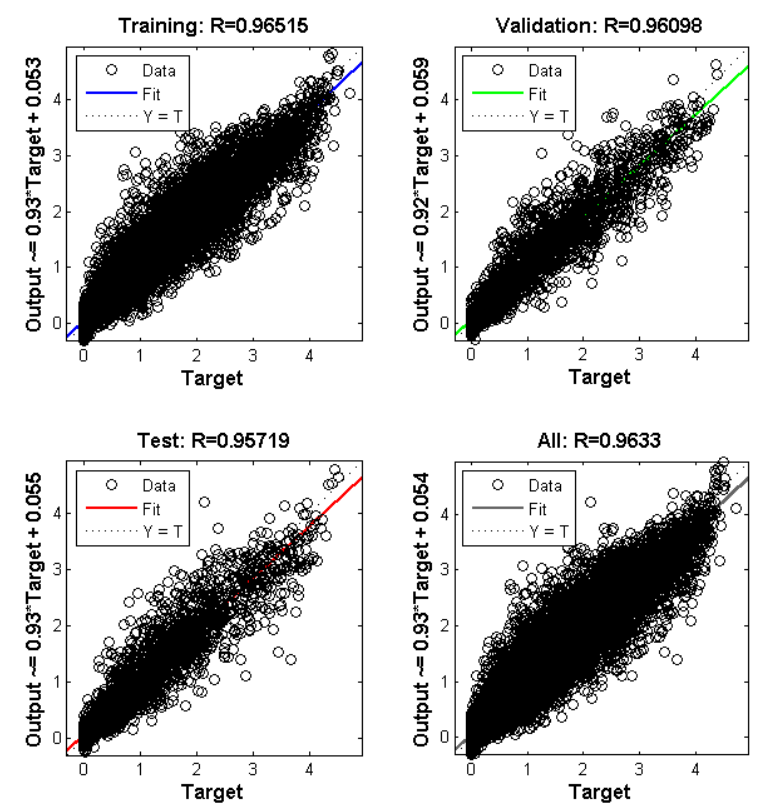

Figure 3: Regression analysis of the network outputs with respect to targets for training,

validation and test sets

For the twelve cases described in Table 1, the NARX network architecture with LM training algorithm was trained, validated and tested. Values of RMSE and regression were closely monitored to find the best approach; Table 4 shows the RMSE and $\mathrm{R}$ values for 90 neurons in the hidden layer.

Table 4: RMSE and Regression values for all 12 NARX networks

\begin{tabular}{|c|c|c|c|c|c|}
\hline Case & RMSE $\left(\mathbf{M J} / \mathbf{m}^{\mathbf{2}}\right)$ & $\mathbf{R}$ & Case & RMSE $\left(\mathbf{M J} / \mathbf{m}^{\mathbf{2}}\right)$ & $\mathbf{R}$ \\
\hline 1 & 0.259 & 0.956 & 7 & 0.270 & 0.953 \\
\hline 2 & $\mathbf{0 . 2 4 3}$ & $\mathbf{0 . 9 6 3}$ & 8 & 0.270 & 0.953 \\
\hline 3 & 0.257 & 0.957 & 9 & 0.259 & 0.956 \\
\hline 4 & 0.266 & 0.953 & 10 & 0.274 & 0.952 \\
\hline 5 & 0.268 & 0.953 & 11 & 0.280 & 0.949 \\
\hline 6 & 0.261 & 0.956 & 12 & 0.277 & 0.950 \\
\hline
\end{tabular}

Figure 4 illustrates this point further, by showing the forecast for an arbitrarily selected single day, that for the first three cases there is correlation between the measured and NARX forecast values for global solar irradiation in Auckland. However, in Table 4, it can be seen that Case 2 is the best among all 12 configurations, with a $0.243 \mathrm{MJ} / \mathrm{m}^{2} \mathrm{RMSE}$ and a 0.963 regression value. 


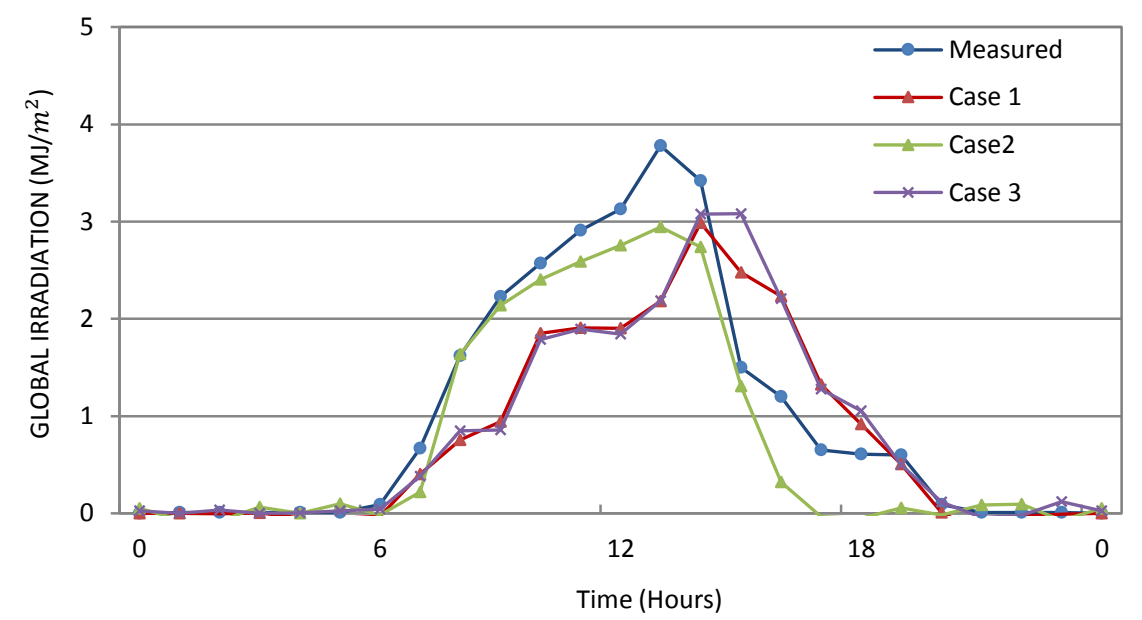

Fig. 4: Measured and predicted irradiation values for Auckland using NARX ANN

Exploring the predictive capability of the NARX architecture further, Figure 5 shows a randomly selected one week forecast of global solar irradiation in Auckland using the weather data from Case 2, presented earlier. It can be seen that over this single week, that the NARX forecasts the solar irradiation with a high degree of accuracy.

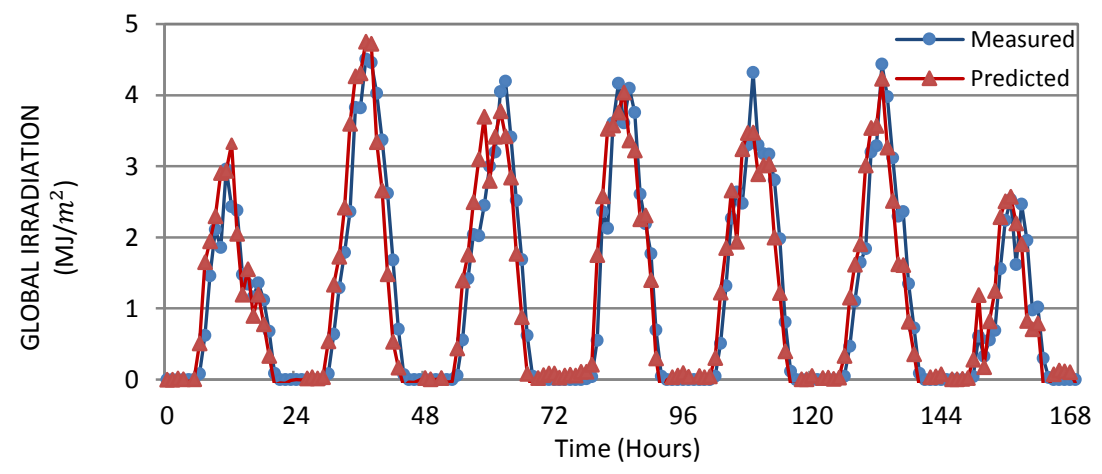

Figure 5. Measured and predicted solar irradiation values using NARX ANN

\subsection{Other forecasting techniques}

Having developed an appropriate NARX ANN forecasting system for Auckland, it was decided to benchmark this approach against three other common forecasting approaches: a Multilayer Perceptron ANN (MLP), an Auto Regressive Moving Average (ARMA) prediction and a persistence forecast to provide a comparative assessment of their forecasting abilities in Auckland. 


\subsubsection{Multilayer perceptron (MLP)}

MLP is a feed forward ANN approach that maps a set of input data onto a set of appropriate output data. An MLP is made of several layers: one input layer, one or several intermediate layers and one output layer as shown in Figure 6. Neurons in input layer only act as buffers for distributing the input signals $x_{i}(i=1,2 \ldots . n)$ to neurons in the hidden layer. As shown in Figure 7, each neuron $j$ in the hidden layer sums up its input signals $X_{i}$ after weighting them with the strengths of the respective connections $w_{i j}$ from the input layer and computes its output $y_{i}$ as a function $f$ of the sum given by Equation 5 .

$$
y_{i}=f\left(\sum_{i=1}^{n} w_{j i} x_{i}\right)
$$

$f$ can be a simple threshold function or a sigmoidal, hyperbolic tangent or radial basis function.

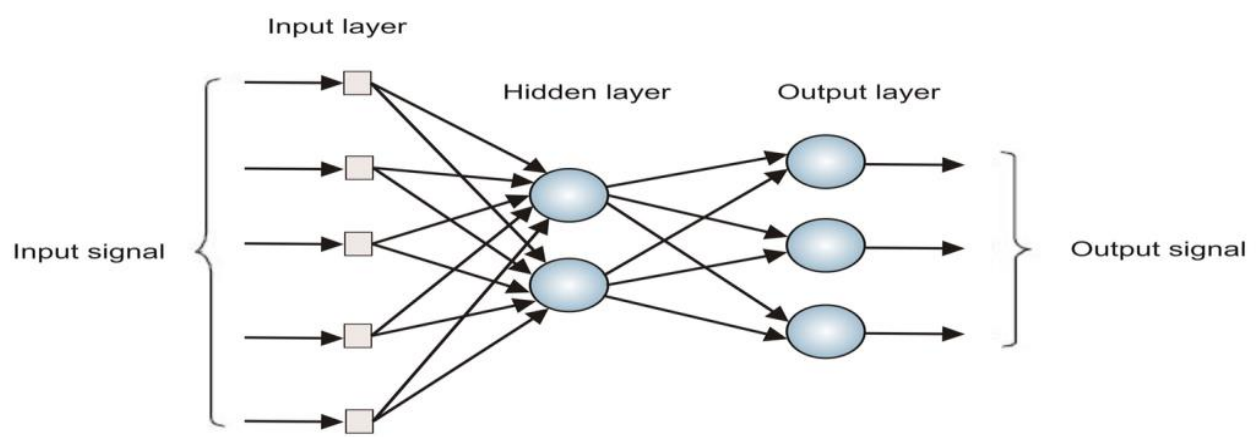

Figure 6. Multi-layered perceptron (MLP) network

In most cases, a single neuron is of no interest however, interconnected single neurons build a network of neurons that can solve complex problems such as classification, pattern recognition and time series prediction. The output of neurons in the output layer is computed similarly and the backpropagation and gradient descent are the most commonly adopted MLP training algorithm. The MLP gives the change $\Delta w_{j i}$ the weight of a connection between neurons $i$ and $j$ as given by Equation 6 .

$$
\Delta w_{j i}=\eta \delta_{j} x_{i}
$$

where $\eta$ is a parameter called the learning rate and $\delta_{j}$ is a factor depending on whether neuron $j$ is an input neuron or a hidden neuron. 


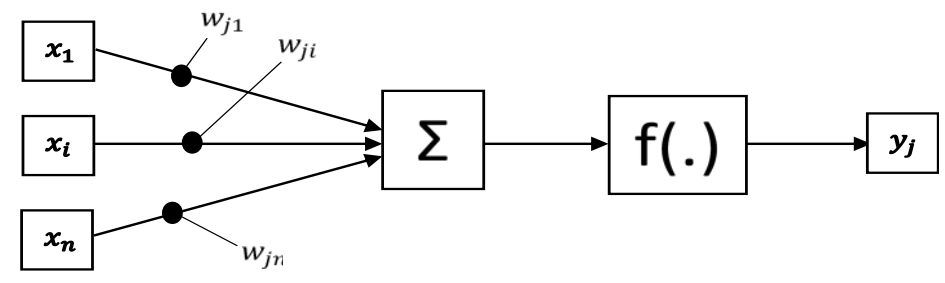

Figure 7.

Detail of the perceptron process

For output neurons, Equation 7 applies, where net $_{j}$ is the total weighted sum of input signals to neurons $j$ and $y_{j}^{(t)}$ is the target output for neuron $j$.

$$
\delta_{j}=\left(\partial_{f} / \partial n e t_{j}\right)\left(y_{j}^{(t)}-y_{j}\right)
$$

And for hidden neurons as there are no target outputs Equation 8 applies, where the difference between the target and actual output of a hidden neurons $j$ is replaced by the weighted sum of the $\delta_{q}$ terms already obtained for neurons $q$ connected to the output of $j$.

$$
\delta_{j}=\left(\partial_{f} / \partial n e t_{j}\right)\left(\Sigma_{q} w_{j q} \delta_{q}\right)
$$

The process begins with the output layer, the $\delta$ term is computed for neurons in all layers and weight updates determined for all connections, iteratively. The weight updating process can happen after the presentation of each training pattern (pattern-based training) or after the presentation of the whole set of training patterns (batch training). Training the epoch is completed when all training patterns have been presented once to the MLP.

A commonly adopted method to speed up the training is to add a "momentum" term to Equation 9, which effectively lets the previous weight change influence the new weight change:

$$
\Delta w_{i j}(I+1)=\eta \delta_{j} x_{i}+\mu \Delta w_{i j}(I)
$$

where $\Delta w_{i j}(I+1)$ and $\Delta w_{i j}(I)$ are weight changes in epochs $(I+I)$ and $(I)$, respectively, and $\mu$ is the "momentum" coefficient (Jayawardena \& Fernando, 1998). Now using the weather data from Case 2, presented earlier, Figure 8 shows a randomly selected one week forecast of global solar radiation in Auckland using the ANN based MLP architecture. RMSE for the ANN based MLP approach was found to be $0.484 \mathrm{MJ} / \mathrm{m}^{2}$ and it can be seen that, in general, the MLP network is able to provide a reasonable forecast of the future irradiation values. 


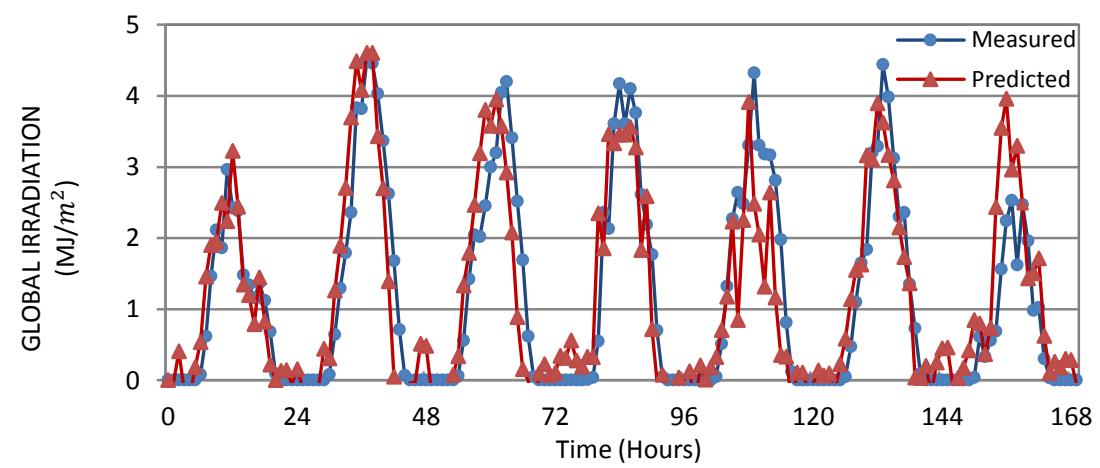

Figure 8. Measured and predicted solar irradiation values using an MLP ANN

\subsubsection{Auto Regressive Moving Average (ARMA)}

ARMA is a type of the time-series analysis that can be used in situations that deal with a large amount of observed data from the past. The ARMA system is developed using Equation Error!

Reference source not found. 0 and consists of two parts, the auto-regressive (AR) part and the moving average (MA) part.

$$
S(t)=\sum_{i=1}^{p} \alpha_{i} S(t-i)+\sum_{j=1}^{q} \beta_{j} e(t-j)
$$

where $S(t)$ is the forecasted solar irradiance at time $t$. In the AR part, $p$ is the order of the AR process, and $\alpha_{i}$ is the AR coefficient. In the MA part, $q$ is the order of the MA error term, $\beta_{j}$ is the MA coefficient and $e(t)$ is the white noise that produces random uncorrelated variables with zero mean and constant variance (Rajagopalan and Santoso 2009). Typically, this method requires large amount of historical data to obtain the ARMA, that is, to find the orders $p, q$ and the coefficients $\alpha_{i}$ and $\beta_{j}$. In addition, due to the geographical differences, each location requires its own unique coefficients. Based on the given historical data, the construction of the ARMA for each location consists of two phases, identifying the orders $p, q$ and determining the coefficients $\alpha_{i}$ and $\beta_{j}$.

The mathematical methods of finding the orders and coefficients of the ARMA architecture are introduced in Torres et al. (2005). The order identification is proposed by Daniel and Chen (1991), and coefficients determination is calculated by applying the Yule-Walker relations for $i$ and the Newton-Raphson algorithms for $j$. 
The ARMA approach was implemented using the MATLAB System Identification Toolbox using the same three years of historical data used for ANN based NARX and MLP approaches. Orders and coefficients for the ARMA model were calculated, as shown in Table 5.

Table 5: Orders and coefficients of the realized ARMA system

\begin{tabular}{|c|c|c|c|}
\hline $\mathbf{p}$ & $\mathbf{q}$ & $\boldsymbol{\alpha}_{\mathbf{i}}$ & $\boldsymbol{\beta}_{\mathbf{j}}$ \\
\hline & & $\boldsymbol{\alpha}_{1}=-\mathbf{1 . 6 6 2}$ & $\boldsymbol{\beta}_{1}=-\mathbf{0 . 3 2 2 3}$ \\
& 3 & $\boldsymbol{\alpha}_{1}=\mathbf{0 . 7 8 5 6}$ & $\boldsymbol{\beta}_{2}=\mathbf{0 . 6 0 7 4}$ \\
& & & $\boldsymbol{\beta}_{3}=0.0591$ \\
\hline
\end{tabular}

Utilising these parameters the global solar irradiance in Auckland was forecast using Equation 11 giving the results for a single week (as used in the MLP forecast) as shown in Figure 9, with a RMSE of $0.315 \mathrm{MJ} / \mathrm{m}^{2}$. From this it can be seen that on the first and seventh day the ARMA forecast values vary considerably from the actual data, while on days 2-6 the forecasted values resemble the actual data. From this it can be suggested that the ARMA approach performs well on a sunny days and its accuracy decreases with increasing clouds cover.

$$
S(t+h)=\sum_{i=1}^{p} \alpha_{i} S(t-i)+\sum_{j=1}^{q} \beta_{j} e(t-j)
$$

where $S(t+h)$ is the forecasted solar irradiance at time $t+h$.

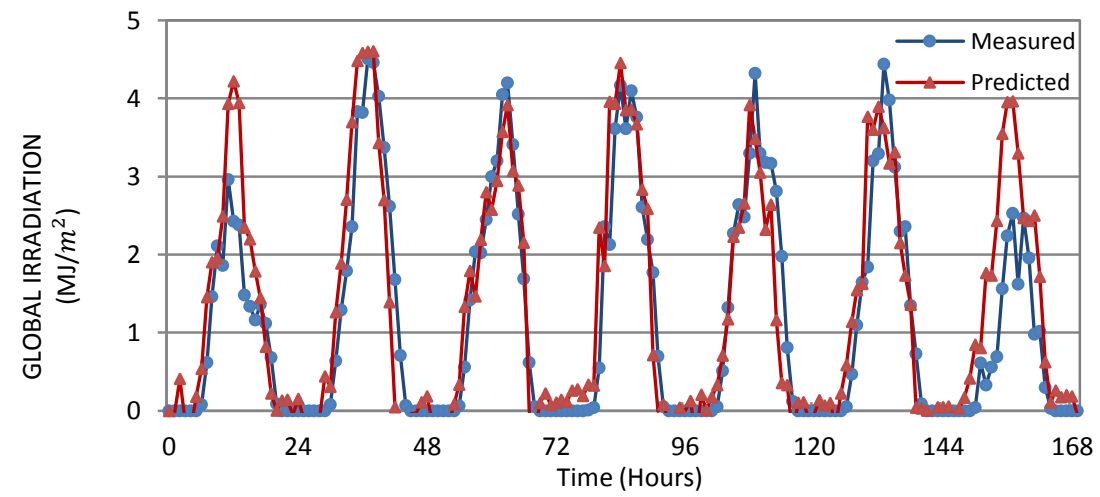

Figure 9. Measured and predicted solar irradiation values using ARMA

\subsubsection{Persistence Forecasting Approach}

As a comparative study, a persistence forecast was developed using Equation 12 to provide the day-ahead forecast ( $h=24$ hours).

$$
S(t+h)=S(t)
$$

where $S(t+h)$ is the forecast solar irradiance at time $t+h$. 
Figure 10 shows a one week forecast of global solar irradiance in Auckland using a persistence approach and the same data used for the MLP and ARMA systems. The average RMSE for the persistence forecast was found to be $0.514 \mathrm{MJ} / \mathrm{m}^{2}$, which was the highest of all the prediction methods discussed. It can be seen in Figure 10 that the persistence model performs well on days $3,4,5$ and 6 whereas the forecasted values for days 1,2, and 7 vary considerably from the actual data.

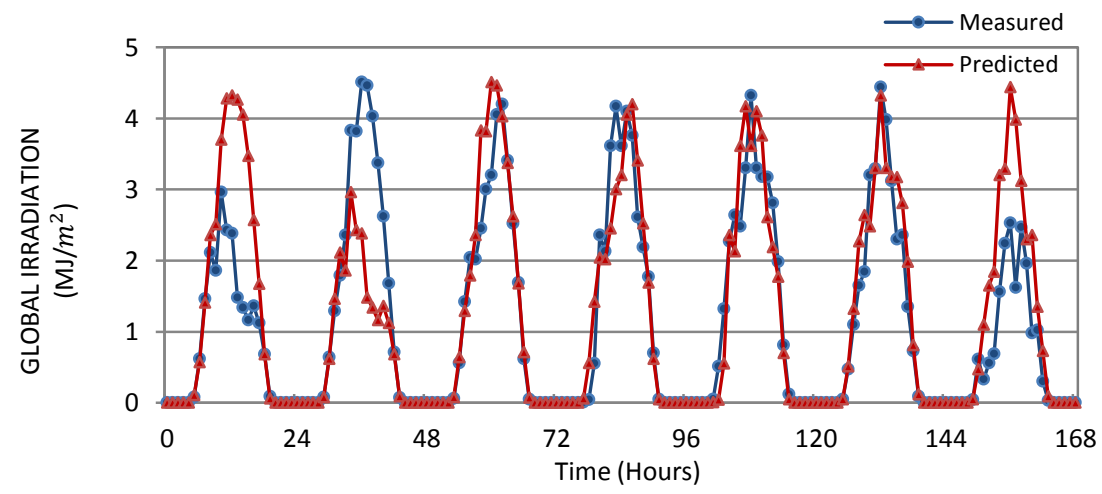

Figure 10. Measured and predicted solar irradiation values using a persistence approach

\section{Results}

\subsection{Comparison of Methods}

In the previous section a discussion of each method was presented and some broad findings were presented, where the same three years of measured data used for the ARMA, MLP and NARX approaches were utilized, with global solar irradiation as the objective function. For each forecast approach the root mean square errors were closely monitored to quantify the performance of the approach and assess their accuracy. Now, Table 6 shows the RMSE and nRMSE values using Equation 4 and 13 respectively for all four approaches, where the same number of hourly data (26363 samples) points for the input variables and the target variable (Global Solar Irradiance) were used to forecast the day ahead solar irradiance in $\mathrm{MJ} / \mathrm{m}^{2}$ for a single year.

$$
\text { nRMSE }=\sqrt{\frac{1}{N} \sum_{i=1}^{N}\left(I_{i}-I_{p}\right)^{2} / I_{i, \text { max }}-I_{i, \min }}
$$

where $I_{p}$ is the predicted solar irradiance in $M J / \mathrm{m}^{2}, I_{i}$ is the measured solar irradiance in $M J / \mathrm{m}^{2}$, and $N$ denotes the number of observations.

Table 6: RMSE and nRMSE values for all forecasting approaches

\begin{tabular}{|l|l|l|} 
Approach & RMSE $\left(\mathbf{M J} / \mathbf{m}^{2}\right)$ & nRMSE $\left(\mathbf{M J} / \mathbf{m}^{2}\right)$ \\
\hline
\end{tabular}




\begin{tabular}{|c|l|l|}
\hline MLP & 0.484 & 0.0968 \\
\hline NARX & 0.243 & 0.0495 \\
\hline ARMA & 0.315 & 0.0656 \\
\hline Persistence & 0.514 & 0.0901 \\
\hline
\end{tabular}

From Table 6 it can be seen that the ARMA approach performs well in terms of the annual RMSE value. Similarly, it was previously demonstrated that the MLP approach performed well for certain days but the RMSE value increases as the number of data sets increases due to the feedforward architecture of the approach, this is borne out by its poor RMSE. In contrast the NARX approach appears to perform well in terms of short term and long term forecasting, and has the lowest annual RMSE of the forecasting approaches tested.

To illustrate this point further, Figure 11 shows an example of the day ahead solar irradiation forecast and measured data curves for all four methods for 1 January 2014 in Auckland. From this it can be seen that the NARX forecast closely follows the actual measurements, whereas the persistence forecast shows a significant under-prediction.

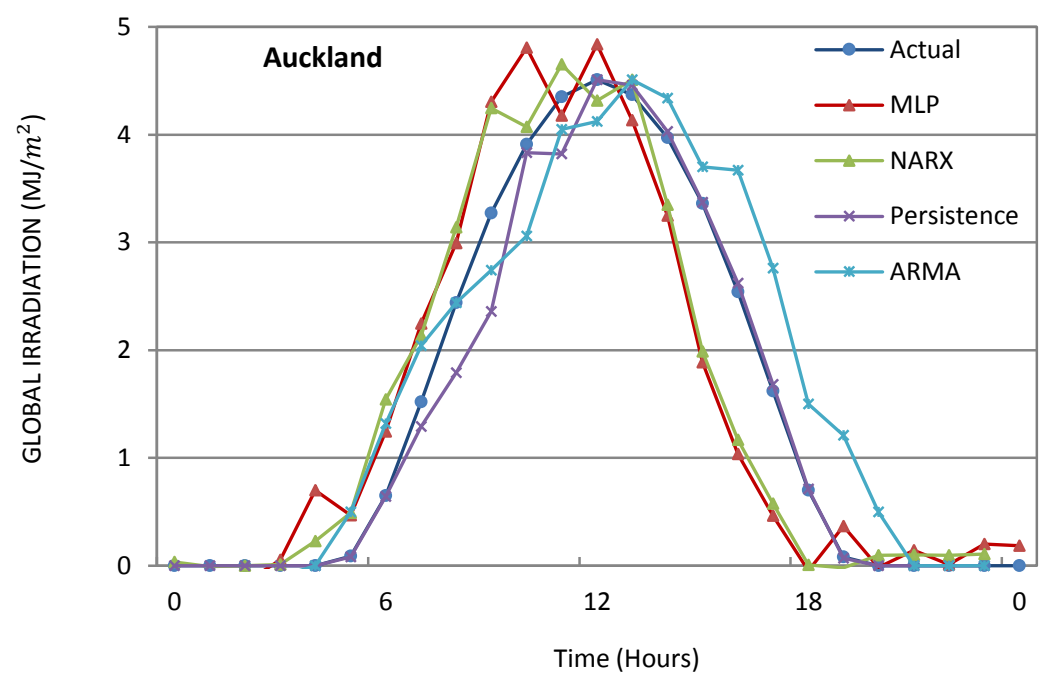

Figure 11. Day ahead solar irradiation forecast and actual data for Auckland on 1 January 2014

\subsection{Irradiation Prediction for New Zealand Cities}

Having determined the most suitable method for forecasting solar irradiation for Auckland, the NARX based Case 2 (as given in Table 2 and trained with data from each location) was used to predict global solar irradiation in ten cities across New Zealand. Figure 12 shows the forecast and measured results for a common single arbitrary day from a one year prediction horizon. Though there is a degree of variation for some locations, as a result of choosing a common single day rather than the best day, it can be seen that using real data to train the ANN gives predicted values 
of global irradiation similar to those measured for the majority of the locations. In this regard, it suggests that recurrent NARX with the LM training algorithm offers a suitable predictive tool for global irradiation in New Zealand. Moreover, it shows that training neural networks with real data can deliver satisfactory prediction of the output variable, in this case the solar irradiation.
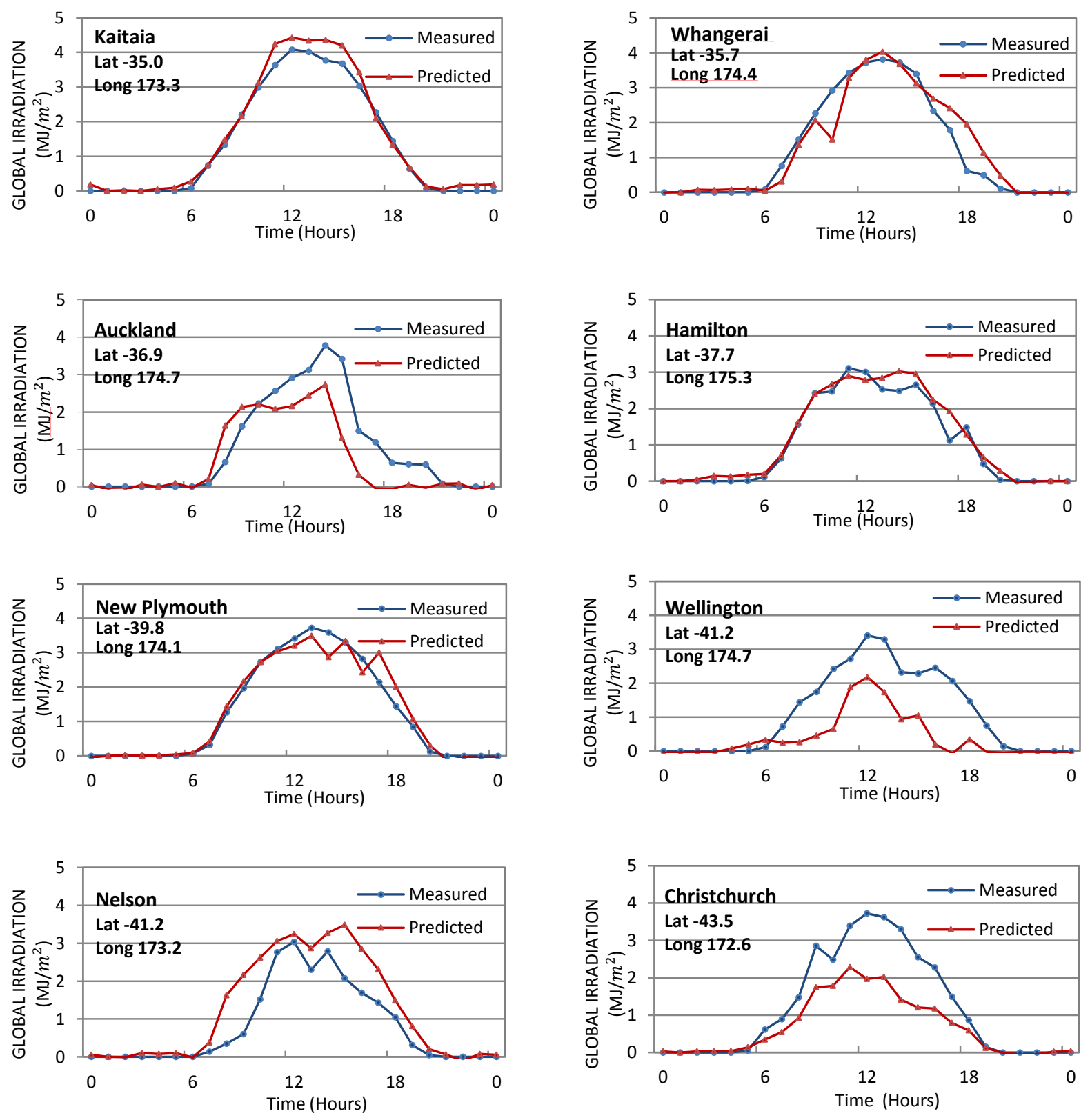

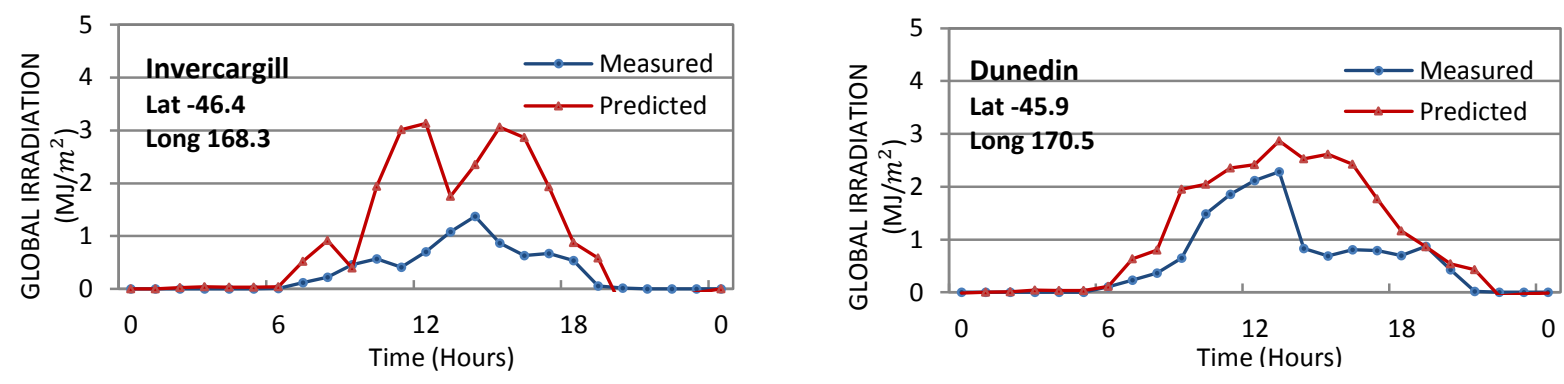

Figure 12: Predicted irradiation for New Zealand cities

\section{Conclusion}

The need for improved solar controllers necessitates the ability of such devices to have an understanding of the future magnitude of the solar resource. This work has examined possible ways in which this can be achieved, with particular reference to a NARX ANN forecasting method. In testing the methods input and target data were used unprocessed to study the real effects of input variables on outputs. Based on an analysis of the root mean squared error, regression and time series response, a NARX approach was proposed as a means to forecast global solar irradiation values at a later time. Subsequently, the NARX architecture was used to successfully forecast global solar irradiation in ten major cities across New Zealand. These results have demonstrated the generalization capability of this approach and its ability to produce accurate forecasts for global irradiation that can be translated to a number of diverse locations. On this basis it is conceivable that such a NARX ANN forecasting approach could be embedded into future solar controllers to better manage the energy generated by solar energy systems.

\section{Acknowledgements}

The authors would like to thank Elaine Fouhy and Ben Liley from NIWA, for their help in collecting weather data for New Zealand. Also thanks to Saeed Ur Rehman, at the University of Auckland and Ian Sumner at Energy Conscious Design Ltd. This work was funded in part by a Callaghan Innovation Education Fellowship.

\section{References}

Al-Alawi, S.M., Al-Hinai, H.A., 1998. An ANN based approach for predicting global solar irradiation in locations with no measurements. Renewable Energy. 14, 199-204. 
Chernichow, T., Piras, A., Imhof, K., Caire, P., Jaccard, Y., Dorizzi, B., Germond, A., 1996. Short term electric load forecasting with artificial neural networks. Engineering Intelligent Systems for Electrical Engineering and Communications. 4, 85-99.

Christophe P., Cyril V., Marc M., Marie-Laure N., 2010. Forecasting of pre-processed daily solar radiation time series using neural networks. Solar Energy. 84, 2146-2160.

Daniel, A., Chen, A., 1991. Stochastic simulation and forecasting of hourly average wind speed sequences in Jamaica. Solar Energy. 46 (1), 1-11.

Fatih, O.H., Omer, N.G., Mehmet, K., 2008. Hourly solar irradiation forecasting using optimal coefficient 2-D linear filters and feed-forward neural network. Solar Energy. 82, 714-726.

Goh, T.N., Tan, K.J., 1977. Stochastic modelling and forecasting of solar irradiation data. Solar Energy. 19, 755-757.

Gueymard, C.A., 2005. Importance of atmospheric turbidity and associated uncertainties in solar irradiation and luminous efficacy modelling. Solar Energy. 30, 1603-1621.

Gueymard, C.A., 2008. Prediction and validation of cloudless shortwave solar spectra incident on horizontal, tilted, or tracking surfaces. Solar Energy. 82, 260-271.

Haykin, S., 1998. Neural networks: a comprehensive foundation, (2nd edition) Prentice Hall, Upper Saddle River, New Jersey.

Esen H., Inalli M., Sengur A., Esen M., 2008. Artificial neural networks and adaptive neuro-fuzzy assessments for ground-coupled heat pump system. Energy and Buildings. 40, 1074-1083.

Jayawardena, A., Achela, D., Fernando, K., 1998. Use of Radial Basis Function type artificial neural networks for Runoff simulation, Computer-Aided Civil and Infrastructure Engineering. 13, $91-99$.

Kalogirou S.A., 2001. Artificial neural networks in energy systems applications: a review. Renewable and Sustainable Energy Reviews. 5, 373-401. 
Loutzenhier P.G., Manz H., Felsmann C., Strachan P.A., Frank T., Maxwell G.M., 2007. Empirical validation of models to compute solar irradiance on inclined surfaces for building energy simulation, Solar Energy. 81, 254-267.

Maitha, H.A., Ali H.A., Hassan A.N., 2011. Using MATLAB to develop artificial neural network Models for predicting Global Solar Irradiation in Al Ain City, UAE, Chapter 9, Engineering Education and Research Using MATLAB, Edited by Dr. Ali Assi, InTech, UAE.

Martin, T.H., Mohammad, B.M., 1994. Training feed-forward networks with the Marquardt Algorithm. IEEE Transactions on Neural Networks. 5, 989-993.

Mellit A., Eleuch H., Benghanem M., Elaoun C., Massi A., 2010. An adaptive model for predicting of global, direct and diffuse hourly solar irradiance. Energy Conversion and Management. 51, 771-782.

Mellit A., Alessandro M. Pavan, 2010. A 24-h forecast of solar irradiance using artificial neural network: Application for performance prediction of a grid-connected PV plant at Trieste, Italy. Solar Energy. 84, 807-821.

Mellit A., Kalogirou S.A., 2008. Artificial intelligence techniques for photovoltaic applications: a review. Progress in Energy and Combustion Science. 34, 574-632.

Mellit A., Kalogirou S.A., Hontoria L., Shaari S., 2009. Artificial intelligence techniques for sizing photovoltaic systems: a review. Renewable and Sustainable Energy Reviews. 13, 406-419.

Moody, J., Utans, J., Rehfuss, S., Siegelmann, H., 1995. Input variable selection for Neural Networks: application to predicting the US business cycle. Computational Intelligence for Financial Engineering, Proceedings of the IEEE/IAFE 1995. 118-122.

Moustrisa K., Paliatsos A.G., Bloutsos A., Nikolaidis K., Koronaki I., Kavadias K., 2008. Use of neural networks for the creation of hourly global and diffuse solar irradiance data at representative locations in Greece. Renewable Energy. 33, 928-932. 
Perez, R., Moore, K., Wilcox, S., Renne, D., Zelenka, A., 2007. Forecasting solar irradiation: preliminary evaluation of an approach based upon the national forecast database. Solar Energy. $81,809-812$.

Rajagopalan, S., Santoso, S., 2009. Wind power forecasting and error analysis using the autoregressive moving average modelling. Power \& Energy Society General Meeting, 2009. PES '09. IEEE, 1- 6, 26-30.

Rich H. Inman., Hugo T.C. Pedro, Carlos F.M. Coimbra, 2013. Solar forecasting methods for renewable energy integration. Progress in Energy and Combustion Science. 39, 535-576.

Sfetsos A., and Coonick A. H., 2000. Univariate and multivariate forecasting of hourly solar radiation with artificial intelligence techniques. Solar Energy. 68, No. 2, 169-178.

National Institute of Water and Atmospheric Research (http://cliflo.niwa.co.nz/) [cited $15 / 04 / 2014]$

Torres, J.L., Garcia, A., De Blas, M., De. Francisco, A., 2005. Forecast of hourly average wind speed with ARMA models in Navarre (Spain). Solar Energy. 79, 65-77.

Ulgen K., Hepbasli A., 2009. Diffuse solar irradiation estimation models for Turkey's big cities. Energy Conversion and Management. 50, 149-156.

Voyant, C., Randimbivololona, P., Nivet, M. L., Paoli, C., Muselli, M., 2014. Twenty four hours ahead global irradiation forecasting using multi-layer perceptron. Meteorological Applications. 21 No. 3, 644-655

Yadav, A.K., Chandel, S.S., 2014. Solar irradiation prediction using artificial neural network techniques: A review. Renewable and Sustainable Energy Reviews. 33, 772-781.

Yousefizadeh, H., Zilouchian, A., 2001. Neural network architectures, Chapter. 3, Intelligent control systems using soft computing methodologies, Edited by A. Zilouchian, M Jamshidi, CRC Press. 
